Supporting information for

\title{
X-ray crystallographic and biochemical characterization of the inhibitory action of an imidazole-dioxolane compound on heme oxygenase
}

Masakazu Sugishima ${ }^{\ddagger}$ Yuichiro Higashimoto ${ }^{\ddagger}$ Tohru Oishi ${ }^{\S}$, Hidenori Takahashi",

Hiroshi Sakamoto ${ }^{\perp}$, Masato Noguchi $i^{\ddagger}$, Keiichi Fukuyama ${ }^{\|, *}$

*Department of Medical Biochemistry, Kurume University School of Medicine, Kurume, Fukuoka 830-0011, Japan, ${ }^{\S}$ Department of Chemistry and "Department of Biological Sciences, Graduate School of Science, Osaka University, Toyonaka, Osaka 560-0043, Japan, and ${ }^{\perp}$ Department of Bioscience and Bioinformatics, Faculty of Computer Science and Systems Engineering, Kyushu Institute of Technology, lizuka, Fukuoka 820-8502, Japan. 


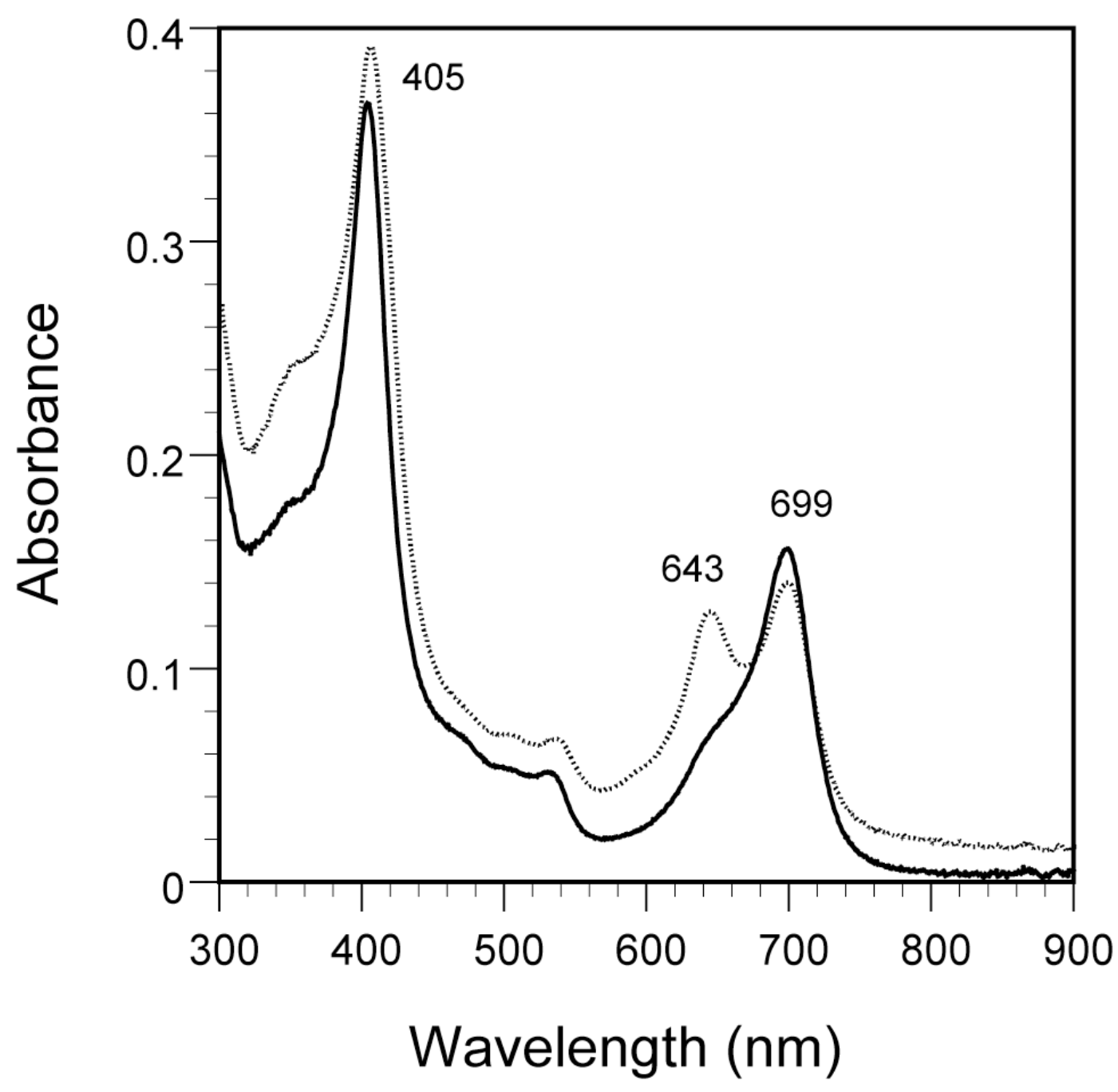

Supporting Figure S1. Spectroscopic characterization of the accumulated intermediates in the NADPH/CPR-supported single-turnover reaction of heme-rat HO-1 complex in the presence of 2-[2-(chlorophenyl)ethyl]-2-[(1H-imidazol-1-yl)methyl]-1,3-dioxolane. The spectra were recorded after the addition of NADPH $(25 \mu \mathrm{M})$ to a solution of the heme-rat HO-1 complex $(5 \mu \mathrm{M})$ and CPR $(0.04 \mu \mathrm{M})$ in $0.1 \mathrm{M}$ potassium phosphate buffer ( $\mathrm{pH} 7.4)$. $\mathrm{CO}$ was added to the reaction mixture $60 \mathrm{~min}$ after the addition of NADPH. Spectra before (- $(-)$ and after $(\cdots . . . . . . . .$.$) the introduction of \mathrm{CO}$ were monitored. 

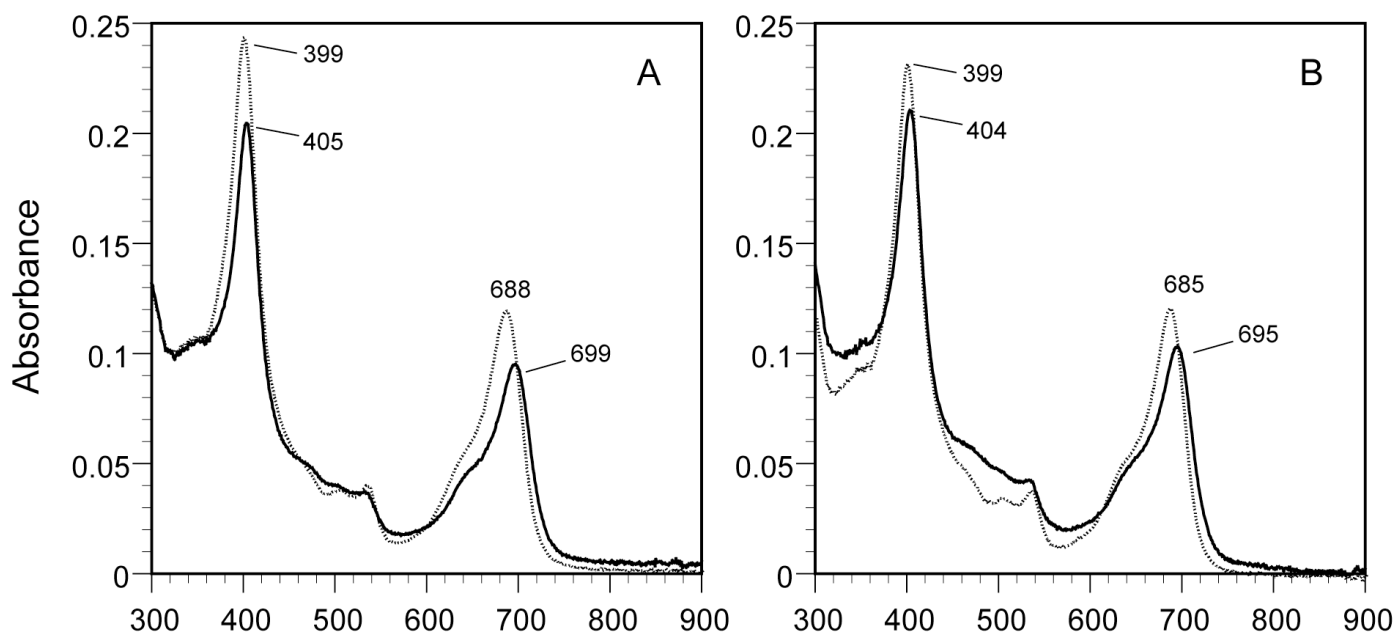

Wavelength $(\mathrm{nm})$

Supporting Figure S2. The spectra of the ferrous verdoheme-rat HO-1 (A) and the ferrous verdoheme-rat HO-2 (B) complexes. The spectra were recorded in the absence (--------) or presence of 2-[2-(4-chlorophenyl)ethyl]-2-[(1H-imidazol-1-yl)methyl]-1,3-dioxolane $\quad(50 \quad \mu \mathrm{M}$, $\longrightarrow$ ). 


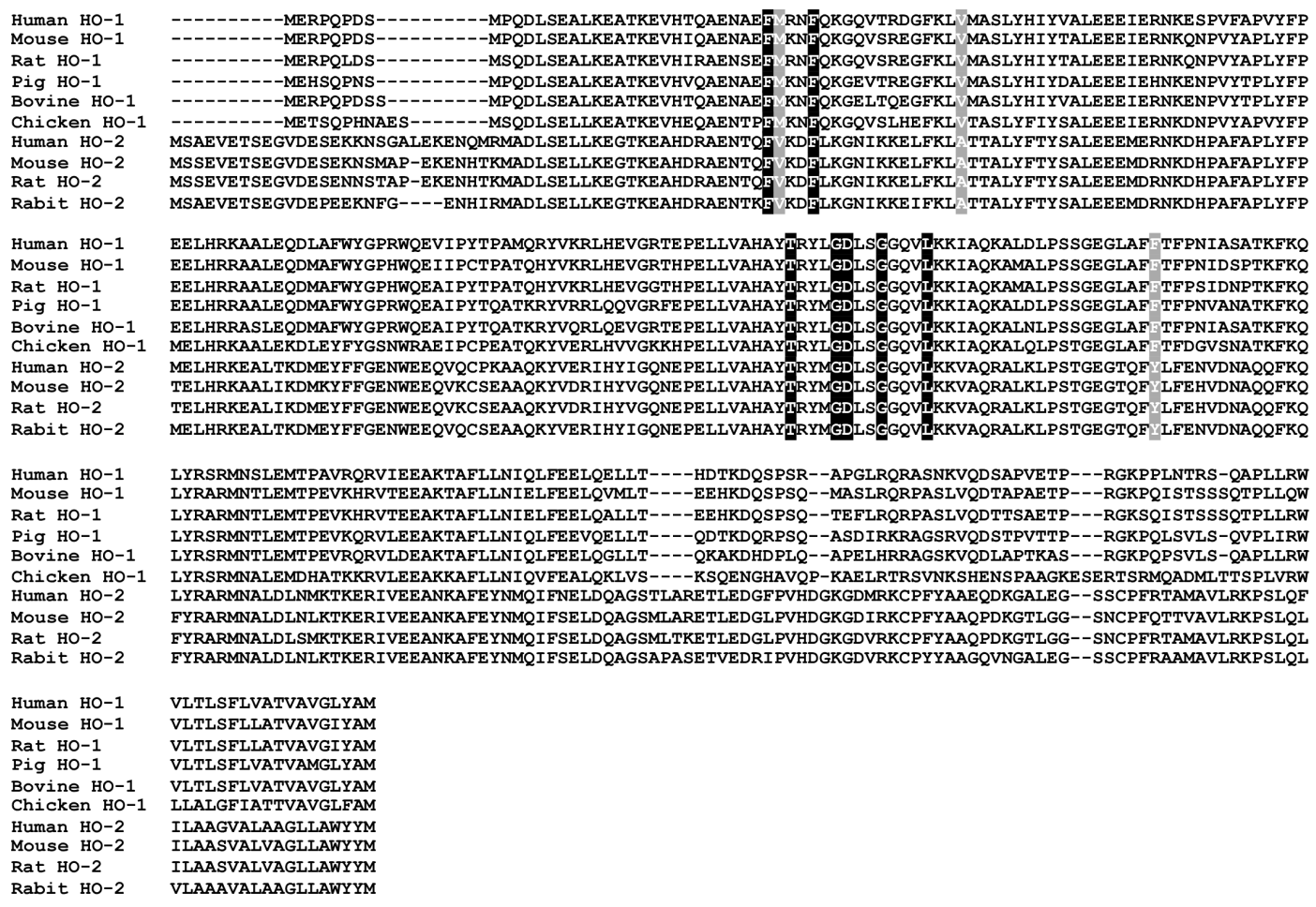

Supporting Figure S3. Sequence alignment of HO-1 and HO-2. Residues involved in the binding of 2-[2-(4-chlorophenyl)ethyl]-2-[(1H-imidazol-1-yl)methyl]-1,3-dioxolane are denoted with white letters on shaded backgrounds. The residues conserved in HO-1 and HO-2 are shown on a black background, whereas those that are not conserved in HO-1 and HO-2 are shown a gray background. The Swissprot accession numbers are P09601 for human HO-1, P14901 for mouse HO-1, P06762 for rat HO-1, P32394 for pig HO-1, Q5E9F2 for bovine HO-1, P14791 for chicken HO-1, P30519 for human HO-2, O70252 for mouse HO-2, $\mathrm{P} 23711$ for rat $\mathrm{HO}-2$, and $\mathrm{P} 43242$ for rabbit $\mathrm{HO}-2$. 